\title{
A Self-Tuning Velocity Observer Formulation for a Class of Nonlinear Systems
}

\author{
Baris Bidikli, Enver Tatlicioglu*, and Erkan Zergeroglu
}

\begin{abstract}
This work presents the design and the corresponding stability analysis of a model free velocity observer formulation for nonlinear systems modeled by Euler-Lagrange formulation. The observation gains of the proposed formulation are tuned online according to an update algorithm removing the burden of observation gain tuning. Lyapunov based arguments are applied to prove the overall system stability. Performance of the observer proposed is illustrated via extensive simulation studies. Experimental studies are also utilized to demonstrate the viability of the proposed formulation.
\end{abstract}

\section{INTRODUCTION}

In this paper, we present a novel velocity observer under the restriction that the system under consideration has dynamical uncertainties. Thus the exact knowledge of dynamical parameters of the system are unavailable for the observer design. Under the standard assumptions that the system dynamics are bounded and first-order differentiable, we proposed a model free velocity observer formulation that achieves asymptotic velocity observation error regulation. In the literature, there are several observer formulations [1], [2], [3], [4], [5], [6], [7] where some of them are modelfree [5], [6], [7]. Different from the available model-free observers, the proposed formulation is self-tuning. That is the observation gains used our formulation are updated online according to an update rule dictated by the stability analysis . To our best knowledge, the self tuning approach used in our formulation is the first presented in the literature. In assistance of the time varying nature of the observer gains, we also were able to remove the requirement of the prior knowledge of the upper bounds of uncertain system dynamics. The stability and convergence properties of the proposed velocity observer are supported by Lyapunovbased arguments. The performance of the designed velocity observer was demonstrated via simulation studies. Experimental studies are also presented to illustrate the effectiveness of the proposed formulation.

B. Bidikli is with the Department of Mechatronics Engineering, The Graduate School of Natural and Applied Sciences, Dokuz Eylul University, Tinaztepe Campus, Buca, Izmir, 35390 Turkey (Email: baris.bidikli@deu.edu.tr).

E. Tatlicioglu is with the Department of Electrical \& Electronics Engineering, Izmir Institute of Technology, Izmir, 35430 Turkey (Phone: +90 (232) 7506536; Fax: +90 (232) 7506599; E-mail: envertatlicioglu@iyte.edu.tr). E. Zergeroglu is with the Department of Computer Engineering, Gebze Institute of Technology, 41400, Gebze, Kocaeli, Turkey (Email: e.zerger@gtu.edu.tr).

E. Tatlicioglu is funded by The Scientific and Technological Research Council of Turkey via grant number $115 \mathrm{E} 726$.

*To whom all the correspondence should be addressed.

\section{System Model ANd Its Properties}

The general model for Euler-Lagrange systems is given as

$$
\ddot{x}=H+G \tau
$$

where $x(t), \dot{x}(t)$ and $\ddot{x}(t) \in \mathbb{R}^{m}$ denote the state vector and its first and second time derivatives, respectively. $H(x, \dot{x}) \in$ $\mathbb{R}^{m}$ and $G(x, \dot{x}) \in \mathbb{R}^{m \times m}$ are nonlinear functions, $\tau(t) \in$ $\mathbb{R}^{m}$ is the control input vector. It is also assumed that the following assumption is satisfied for the model in (1).

Assumption 1: $H(x, \dot{x})$ and $G(x, \dot{x})$ are $\mathcal{C}^{1}$ functions. The control input is a $\mathcal{C}^{1}$ function and $\tau(t), \dot{\tau}(t) \in \mathcal{L}_{\infty}$. The system states are bounded for all time (i.e., $x(t), \dot{x}(t) \in$ $\left.\mathcal{L}_{\infty}\right)$.

Remark 1: The mathematical model for an $m$ degree-offreedom, revolute joint, direct drive, robot manipulator is given as [8]

$$
M(q) \ddot{q}+V_{m}(q, \dot{q}) \dot{q}+g(q)+F_{d} \dot{q}=\tau
$$

where $q(t), \dot{q}(t)$ and $\ddot{q}(t) \in \mathbb{R}^{m}$ denote the link position, velocity and acceleration, respectively, $M(q) \in \mathbb{R}^{m \times m}$ represents the positive-definite, symmetric inertia matrix, $V_{m}(q, \dot{q}) \in \mathbb{R}^{m \times m}$ represents the centripetal Coriolis matrix, $g(q) \in \mathbb{R}^{m}$ is the gravitational vector, $F_{d} \in \mathbb{R}^{m \times m}$ denotes the constant, diagonal positive-definite, viscous friction matrix, and $\tau(t)$ being the control input torque, can be rearranged as a model that is given in (1) by selecting $x \triangleq q$, $H \triangleq-M^{-1}\left(V_{m} \dot{q}+g+F_{d} \dot{q}\right)$ and $G \triangleq M^{-1}$.

\section{OBSERVER DESIGN}

Estimating the unavailable velocity signal $\dot{x}(t)$ by designing an observer having online gain tuning methodology is the main objective of this study. We realize our design under the restriction that $H(x, \dot{x}), G(x, \dot{x})$ and $\tau(t)$ are unavailable. To quantify this objective, the velocity observation error denoted by $\dot{\tilde{x}}(t) \in \mathbb{R}^{m}$ is defined as

$$
\dot{\tilde{x}} \triangleq \dot{x}-\dot{\hat{x}}
$$

where $\dot{\hat{x}}(t) \in \mathbb{R}^{m}$ is the observed velocity. In view of (3), the position observation error, $\tilde{x}(t) \in \mathbb{R}^{m}$, is defined in the following manner

$$
\tilde{x} \triangleq x-\hat{x} .
$$

The velocity observer is designed in the following form

$$
\dot{\hat{x}}=p+\left[k(t)+I_{m}\right] \tilde{x}
$$


where $I_{m} \in \mathbb{R}^{m \times m}$ is the standard identity matrix while the auxiliary variable $p(t) \in \mathbb{R}^{m}$ is updated according to following rule

$$
\dot{p}=\hat{\beta}(t) \operatorname{Sgn}(\tilde{x})+k(t) \tilde{x} .
$$

In (5) and (6), $\hat{\beta}(t)$ and $k(t) \in \mathbb{R}^{m \times m}$ denote diagonal time varying gain matrices while $\operatorname{Sgn}(\cdot)$ is the vector signum function. The diagonal entries of time varying gain $k(t)$ are updated according to

$$
k_{i}(t)=1+k_{c_{i}}+\frac{1}{2} \tilde{x}_{i}^{2}(t)+\int_{t_{0}}^{t} \tilde{x}_{i}^{2}(\sigma) d \sigma
$$

where $k_{c_{i}} \in \mathbb{R}$ being entries of the positive-definite, diagonal constant part of $k(t)$ denoted by $k_{c}(t) \in \mathbb{R}^{m \times m}$ and $\tilde{x}_{i}(t) \in$ $\mathbb{R}$ is the $i^{t h}$ entry of $\tilde{x}(t)$. The other time varying gain $\hat{\beta}(t)$ has the following structure

$$
\hat{\beta}(t)=\hat{\beta}_{1}(t)+\beta_{2}
$$

where $\hat{\beta}_{1}(t) \in \mathbb{R}^{m \times m}$ is its diagonal time varying part while $\beta_{2} \in \mathbb{R}^{m \times m}$ being its positive definite, diagonal, constant part. The diagonal entries of the time varying part of the control gains are updated according to

$$
\hat{\beta}_{1_{i}}(t)=\left|\tilde{x}_{i}(t)\right|-\left|\tilde{x}_{i}\left(t_{0}\right)\right|+\int_{t_{0}}^{t}\left|\tilde{x}_{i}(\sigma)\right| d \sigma
$$

where $\hat{\beta}_{1_{i}}\left(t_{0}\right)=0$. Taking the time derivative of (5) yields

$$
\ddot{\hat{x}}=\hat{\beta}(t) \operatorname{Sgn}(\tilde{x})+[k(t)+\dot{k}(t)] \tilde{x}+\left[k(t)+I_{m}\right] \dot{\tilde{x}}
$$

where (6) was substituted. Subtracting (10) from (1), the velocity observation error dynamics is obtained as

$$
\begin{aligned}
\ddot{\tilde{x}}= & H+G \tau-\hat{\beta}(t) \operatorname{Sgn}(\tilde{x})-[k(t)+\dot{k}(t)] \tilde{x} \\
& -\left[k(t)+I_{m}\right] \dot{\tilde{x}} .
\end{aligned}
$$

Let the auxiliary error signal $r(t) \in \mathbb{R}^{m}$ is defined as

$$
r \triangleq \dot{\tilde{x}}+\tilde{x}
$$

We would like to note that from the definition of $r(t)$ given above, as $r(t)$ requires $\dot{x}(t)$ it cannot be utilized directly in the observer design. Substituting (11) into the time derivative of (12), we obtain

$$
\begin{aligned}
\dot{r}= & N(x, \dot{x}, t)-\left[\hat{\beta}_{1}(t)+\beta_{2}\right] \operatorname{Sgn}(\tilde{x}) \\
& -k(t) r-\dot{k}(t) \tilde{x}
\end{aligned}
$$

where (8) was utilized and the auxiliary function $N(x, \dot{x}, t) \in \mathbb{R}^{m}$ is defined as

$$
N \triangleq H+G \tau
$$

At this point, it should be stated that as a consequence of Assumption 1 the functions $N$ and $\dot{N}$ are bounded functions of time.

\section{Stability Analysis}

In this section, the stability of the velocity observation error is investigated. Firstly, two lemmas are stated where both of which will later be utilized in the proof of the main theorem.

Lemma 1: The auxiliary function, denoted by $L_{1}(t) \in \mathbb{R}$, is defined as

$$
L_{1} \triangleq r^{T}\left[N-\beta_{1} \operatorname{Sgn}(\tilde{x})\right]
$$

where $\beta_{1} \in \mathbb{R}^{m \times m}$ is a positive definite, diagonal constant term. Provided that the entries of $\beta_{1}$ satisfy

$$
\beta_{1_{i}} \geq\left\|N_{i}(t)\right\|_{L_{\infty}}+\left\|\dot{N}_{i}(t)\right\|_{L_{\infty}}
$$

where $\|\cdot\|_{L_{\infty}}$ denotes infinity norm, then

$$
\int_{t_{0}}^{t} L_{1}(\sigma) \leq \zeta_{b_{1}}
$$

where $\zeta_{b_{1}} \in \mathbb{R}$ is a positive constant.

Remark 2: Notice that the constant matrix denoted by $\beta_{1}$ needs to satisfy the condition in (16) and an observer formulation using $\beta_{1}$ would require at least the prior knowledge of the upper bounds of the system uncertainties. However, the observer proposed in (5) and (6), does not make use of $\beta_{1}$ but $\hat{\beta}_{1}$. Thus does not require the bounds of the functions containing the uncertainties of the dynamics.

Proof: See Appendix I for the proof.

Lemma 2: The auxiliary function, denoted by $L_{2}(t) \in \mathbb{R}$, is defined as

$$
L_{2} \triangleq-\dot{\tilde{x}}^{T} \beta_{2} \operatorname{Sgn}(\tilde{x})
$$

Provided that $\beta_{2_{i}}>0$ for $i=1, \cdots, m$ then

$$
\int_{t_{0}}^{t} L_{2}(\sigma) d \sigma \leq \zeta_{b_{2}}
$$

where $\zeta_{b_{2}} \in \mathbb{R}$ is a positive constant.

Proof: See Appendix II for the proof.

Theorem 1: The observer in (5) and (6) with the time varying gains in (7)-(9) ensures global asymptotic convergence of the velocity observation error in the sense that $\|\dot{\tilde{x}}(t)\| \rightarrow 0$ as $t \rightarrow \infty$.

Proof: The Lyapunov function candidate, denoted by $V(y, t) \in \mathbb{R}$, is defined as

$$
V \triangleq \frac{1}{2} r^{T} r+\frac{1}{2} \sum_{i=1}^{m} \tilde{\beta}_{1_{i}}^{2}+P_{1}+P_{2}
$$

where $P_{1}(t), P_{2}(t) \in \mathbb{R}$ are defined as

$$
\begin{aligned}
& P_{1} \triangleq \zeta_{b_{1}}-\int_{t_{0}}^{t} L_{1}(\sigma) d \sigma \\
& P_{2} \triangleq \zeta_{b_{2}}-\int_{t_{0}}^{t} L_{2}(\sigma) d \sigma
\end{aligned}
$$

and $\tilde{\beta}_{1_{i}}(t) \in \mathbb{R}, i=1, \cdots, m$ are defined as

$$
\tilde{\beta}_{1_{i}} \triangleq \beta_{1_{i}}-\hat{\beta}_{1_{i}}
$$


and $y(t) \in \mathbb{R}^{(2 m+2)}$ is defined as

$$
y \triangleq\left[\begin{array}{llllll}
r^{T} & \tilde{\beta}_{1_{1}} & \cdots & \tilde{\beta}_{1_{m}} & \sqrt{P}_{1} & \sqrt{P}_{2}
\end{array}\right]^{T} .
$$

From the proofs of Lemmas 1 and 2, we can conclude that $P_{1}(t)$ and $P_{2}(t)$ are non-negative and thus $V(y, t)$ is also non-negative.

After taking the time derivative of (20) and substituting the time derivative of (9) and (13), following expression can be obtained

$$
\dot{V}=-r^{T} k r-\sum_{i=1}^{m} \beta_{2_{i}}\left|\tilde{x}_{i}\right|-r^{T} \dot{k} \tilde{x}
$$

where (15) and (18) were utilized. An upper bound on (25) can be obtained as

$$
\dot{V} \leq-r^{T} k r-\sum_{i=1}^{m} \beta_{2_{i}}\left|\tilde{x}_{i}\right|-\sum_{i=1}^{m} r_{i}^{2} \tilde{x}_{i}^{2}
$$

where the time derivative of (7) was utilized along with (12). From (26), it can be stated that

$$
\dot{V} \leq-\sum_{i=1}^{m} k_{c_{i}} r_{i}^{2}-\sum_{i=1}^{m} \beta_{2_{i}}\left|\tilde{x}_{i}\right|
$$

where (7) was utilized. From (20) and (27), we can state that $V(y, t) \in \mathcal{L}_{\infty}$ and thus $r(t), \tilde{\beta}_{1_{i}}(t)$ for $i=1, \cdots, m$, $P_{1}(t), P_{2}(t) \in \mathcal{L}_{\infty}$. Boundedness of $r(t)$ can be utilized along with (12) to show that $\tilde{x}(t)$ and $\dot{\tilde{x}}(t) \in \mathcal{L}_{\infty}$. From (3), (4), Assumption 1 and $x(t), \dot{\tilde{x}}(t) \in \mathcal{L}_{\infty}$ it can be concluded that $\hat{x}(t)$ and $\dot{\hat{x}}(t) \in \mathcal{L}_{\infty}$. Integrating (27) we obtain,

$$
\begin{array}{r}
\sum_{i=1}^{m} k_{c_{i}} \int_{t_{0}}^{\infty} r_{i}^{2}(\sigma) d \sigma+\sum_{i=1}^{m} \beta_{2_{i}} \int_{t_{0}}^{\infty}\left|\tilde{x}_{i}(\sigma)\right| d \sigma \leq \\
V\left(t_{0}\right)-V(\infty)
\end{array}
$$

and since $V(\infty) \geq 0$ following expressions are valid

$$
\begin{array}{r}
\sum_{i=1}^{m} k_{c_{i}} \int_{t_{0}}^{\infty} r_{i}^{2}(\sigma) d \sigma \leq V\left(t_{0}\right) \\
\text { and } \\
\sum_{i=1}^{m} \beta_{2_{i}} \int_{t_{0}}^{\infty}\left|\tilde{x}_{i}(\sigma)\right| d \sigma \leq V\left(t_{0}\right), i=1, \cdots, m .
\end{array}
$$

From (29), we can claim that $r(t) \in \mathcal{L}_{2}$ and $\tilde{x}(t) \in \mathcal{L}_{1}$. Since $\tilde{x}(t) \in \mathcal{L}_{1} \cap \mathcal{L}_{\infty}$, from (9), it is concluded that $\hat{\beta}_{1}(t) \in \mathcal{L}_{\infty}$, and since $r(t) \in \mathcal{L}_{\infty}$, then from the derivative of (9), $\dot{\hat{\beta}}_{1}(t) \in \mathcal{L}_{\infty}$. From the above boundedness statements and (7), it is clear that $k(t) \in \mathcal{L}_{\infty}$. Standard signal chasing arguments can be utilized to prove that all the remaining signals remain bounded under the closed-loop operation. Since $r(t) \in \mathcal{L}_{2} \cap \mathcal{L}_{\infty}$ and $\dot{r}(t) \in \mathcal{L}_{\infty}$, Barbalat's Lemma [9] can be utilized to prove that $\|r(t)\| \rightarrow 0$ as $t \rightarrow \infty$, and from (12), we can show that the velocity observation error converges asymptotically to zero.

Theorem 2: There exist constants $\hat{\beta}_{i_{f}}$ and $k_{i_{f}} \in \mathbb{R}$ such that

$$
\hat{\beta}_{i}(t) \rightarrow \hat{\beta}_{i_{f}} \text { and } k_{i}(t) \rightarrow k_{i_{f}} \text { as } t \rightarrow \infty .
$$

Proof: Applying the limit operation to (9) yields

$$
\lim _{t \rightarrow \infty} \hat{\beta}_{1_{i}}(t)=-\left|\tilde{x}_{i}\left(t_{0}\right)\right|+\lim _{t \rightarrow \infty}\left[\int_{t_{0}}^{t}\left|\tilde{x}_{i}(\theta)\right| d \theta\right]
$$

where $\tilde{x}_{i}(t) \rightarrow 0$ was utilized. Since, from the proof of Theorem 1, $\tilde{x}_{i}(t) \in \mathcal{L}_{1}$, the existence of $\lim _{t \rightarrow \infty}\left[\int_{t_{0}}^{t}\left|\tilde{x}_{i}(\theta)\right| d \theta\right]$ is ensured via Theorem 3.1 of [10]. In view of this, from (31), it is straight forward to see that (30) is obtained.

Similarly, taking the limit of (7) results in

$$
\lim _{t \rightarrow \infty} k_{i}(t)=k_{c_{i}}+\lim _{t \rightarrow \infty}\left[\int_{t_{0}}^{t} \tilde{x}_{i}^{2}(\theta) d \theta\right]
$$

where $\tilde{x}_{i}(t) \rightarrow 0$ was utilized. Since $\tilde{x}_{i}(t) \in \mathcal{L}_{2}$, from Theorem 3.1 in [10], the existence of $\lim _{t \rightarrow \infty}\left[\int_{t_{0}}^{t} \tilde{x}_{i}^{2}(\theta) d \theta\right]$ is ensured from which the result in (30) follows.

\section{Simulation Study}

The two link, direct-drive planar robot manipulator having the following dynamic model was used in the numerical simulations [11]

$$
\begin{aligned}
{\left[\begin{array}{l}
\tau_{1} \\
\tau_{2}
\end{array}\right]=} & {\left[\begin{array}{ll}
p_{1}+2 p_{3} c_{2} & p_{2}+p_{3} c_{2} \\
p_{2}+p_{3} c_{2} & p_{2}
\end{array}\right]\left[\begin{array}{l}
\ddot{q}_{1} \\
\ddot{q}_{2}
\end{array}\right] } \\
+ & {\left[\begin{array}{ll}
-p_{3} s_{2} \dot{q}_{2} & -p_{3} s_{2}\left(\dot{q}_{1}+\dot{q}_{2}\right) \\
p_{3} s_{2} \dot{q}_{1} & 0
\end{array}\right]\left[\begin{array}{l}
\dot{q}_{1} \\
\dot{q}_{2}
\end{array}\right] } \\
+ & {\left[\begin{array}{ll}
f_{d 1} & 0 \\
0 & f_{d 2}
\end{array}\right]\left[\begin{array}{l}
\dot{q}_{1} \\
\dot{q}_{2}
\end{array}\right] }
\end{aligned}
$$

where $p_{1}=3.473\left[k g-m^{2}\right], p_{2}=0.193\left[k g-m^{2}\right]$, $p_{3}=0.242\left[\mathrm{~kg}-\mathrm{m}^{2}\right], f_{d 1}=5.3[\mathrm{Nm}-\mathrm{sec}], f_{d 2}=$ $1.1[N m-s e c], c_{2} \triangleq \cos \left(q_{2}\right)$, and $s_{2} \triangleq \sin \left(q_{2}\right)$. During these simulations a full state feedback control approach that had been designed in [12] was used as a controller with the control objective that force $q_{1}(t)$ and $q_{2}(t)$ follow a sinusoidal desired trajectory denoted by $q_{r}(t) \in \mathbb{R}^{2}$ and given as

$$
q_{r}(t)=\left[\begin{array}{c}
0.7 \sin (t)\left(1-\exp \left(-0.3 t^{3}\right)\right) \\
1.2 \sin (t)\left(1-\exp \left(-0.3 t^{3}\right)\right)
\end{array}\right] .
$$

Constant part of the observation gains were simply selected as $k_{c}=\beta_{2}=I_{2}$. The results are shown in Figures 1-5. The observed velocity is shown in Figure 1. The observation errors are shown in Figure 2, while the observed and the actual positions are depicted in Figure 3. From Figures 2 and 3 , it is clear that the observation objective was met. The time varying gains $\hat{\beta}(t)$ and $k(t)$ are shown in Figures 4 and 5 , respectively. From these figures, it can clearly be seen that the time varying gains were converged to constant values.

\section{EXPERIMENTAL STUDY}

PHANToM Omni haptic device that is shown in Figure 6 was used as an experimental setup. During the experimental studies the first link of this device was mechanically stopped and the observation was realized for the remaining links while their joint space control was realized via same full 

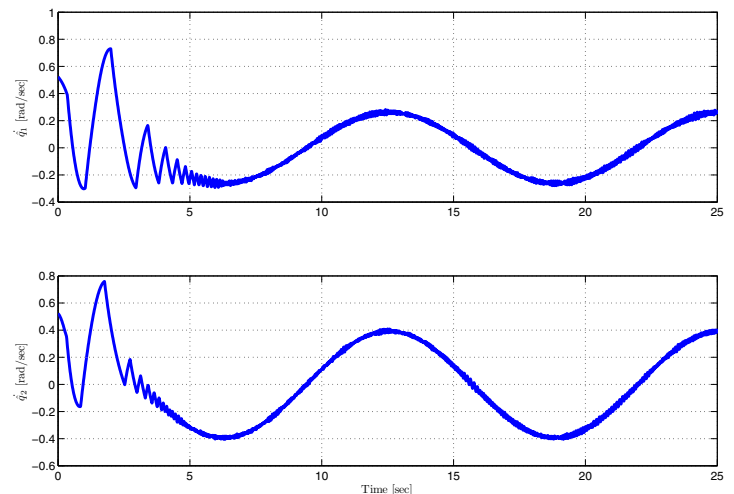

Fig. 1. Observed velocities $\dot{\hat{q}}(t)$
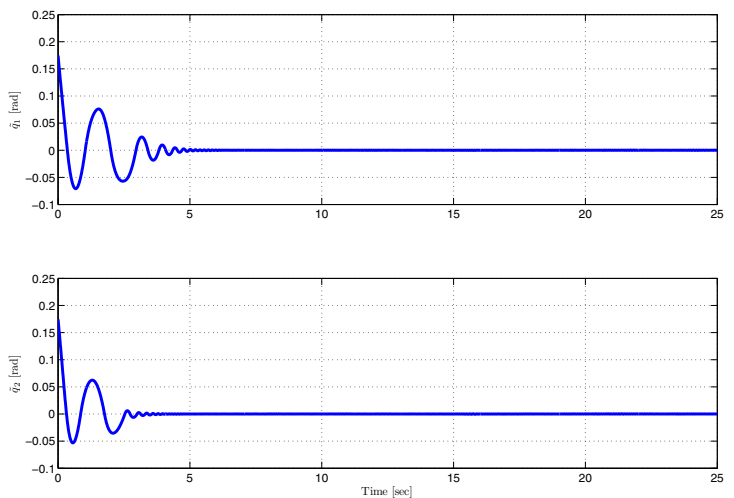

Fig. 2. Position observation errors $\tilde{q}(t)$

state feedback control approach to obtain the consistency with simulation studies. During the experimental studies, same control approach with simulation studies was used with the control objective that is to make $\theta_{2}(t)$ and $\theta_{3}(t)$ follow a sinusoidal desired trajectory denoted by $\theta_{r}(t) \in \mathbb{R}^{2}$ and given as

$$
\theta_{r}(t)=\left[\begin{array}{l}
0.5+0.1 \sin (0.1 t) \\
\cos (0.1 t)
\end{array}\right] .
$$

Constant parts of the observation gains were selected as $k_{c}=$ $\beta_{2}=I_{2}$.

The results are shown in Figures 7-11. The observed velocity is shown in Figure 7. The observation errors are shown in Figure 8, while the observed and the actual positions are depicted in Figure 9. From Figures 8 and 9, it is clear that the observation objective was met. The change of the time varying gains $\hat{\beta}(t)$ and $k(t)$ are shown in Figures 10 and 11 , respectively. From these figures, it can clearly be seen that the time varying gains are converged to their constant values.

\section{CONCLUSIONS}

In this paper, we have presented a novel model-free velocity observer with time varying observation gains. The proposed formulation does not need a tuning methodology
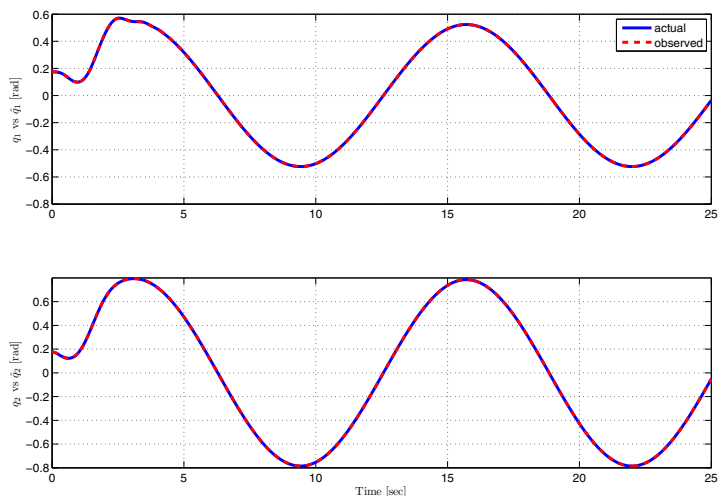

Fig. 3. Actual positions $q(t)$ (line) vs observed positions $\hat{q}(t)$ (dashed)
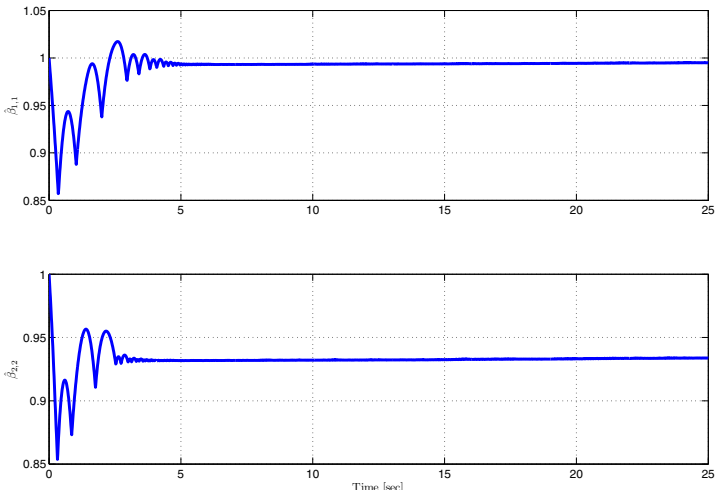

Fig. 4. Diagonal entries of $\hat{\beta}(t)$

and does not require prior knowledge of upper bounds of the vector containing uncertainties for the observer gain selection. The observer formulation achieved global observation and the stability result is backed up with a Lyapunovtype analysis. Extensive simulation studies is presented to illustrate the observation performance of the proposed method. The observation performance is also demonstrated by utilizing experimental studies. When compared with the existing velocity observers in the literature, according to the authors' best knowledge the results in this paper are the only design that obtained observation gains with a self-tuning methodology. Thus, ensuring the global asymptotic velocity observation for Euler-Lagrange systems by getting rid of the gain adjusting process can be considered as the main novelty of this study.

\section{REFERENCES}

[1] S. Nicosia and P. Tomei, "Robot control by using only joint position measurements," IEEE Tr. on Automatic Control, vol. 35, no. 9, pp. 1058-1061, 1990

[2] M. Erlic and W. Lu, "Manipulator control with an exponentially stable velcity observer," in Proc. American Control Conf., Chicago, IL, USA, 1992, pp. 1241-1242.

[3] H. Berghuis and H. Nijmeijer, "A passivity approach to controllerobserver design for robots," IEEE Tr. on Robotics and Automation, vol. 9, no. 6, pp. 740-754, 1993. 

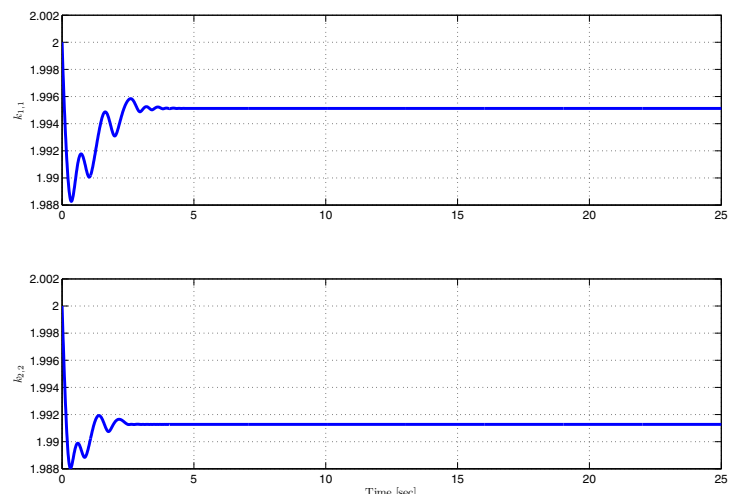

Fig. 5. Diagonal entries of $k(t)$

Fig. 6. PHANToM Omni Haptic Device

[4] S. Y. Lim, D. M. Dawson, and K. Anderson, "Re-examining the Nicosia-Tomei robot observer-controller from a backstepping perspective," IEEE Tr. on Control System Technology, vol. 4, no. 3, pp. 304-310, 1996.

[5] S. Arimoto, V. Parra-Vega, and T. Naniwa, "A class of linear velocity observers for nonlinear mechanical systems," in Proc. Asian Control Conference, Tokyo, Japan, 1994, pp. 633-636.

[6] B. Xian, M. S. de Queiroz, D. M. Dawson, and M. L. McIntyre, "A discontinuous output feedback controller and velocity observer for nonlinear mechatronic systems," Automatica, vol. 40, no. 4, pp. 695700, 2004.

[7] A. Bayrak, E. Tatlicioglu, E. Zergeroglu, and M. Deniz, "A new continuous velocity observer formulation for a class of uncertain nonlinear mechanical systems," in Proc. Int. Conf. on Control Applications, Sydney, Australia, 2010, pp. 602-607.

[8] F. L. Lewis, C. T. Abdallah, and D. M. Dawson, Control of Robot Manipulators. New York, NY, USA: Macmillan Publishing Co., 1993.

[9] M. Krstic, I. Kanellakopoulos, and P. Kokotovic, Nonlinear and Adaptive Control Design. New York, NY, USA: John Wiley \& Sons, 1995.

[10] M. Krstic, "Invariant manifolds and asymptotic properties of adaptive nonlinear stabilizers," IEEE Trans. Autom. Control, vol. 41, no. 6, pp. 817-829, 1996.

[11] E. Zergeroglu, D. M. Dawson, M. S. de Queiroz, and M. Krstic, "On global output feedback tracking control of robot manipulators," in Proc. IEEE Int. Conf. Decision and Control, Sydney, Australia, 2015, pp. 1278-1283.

[12] B. Bidikli, E. Tatlicioglu, E. Zergeroglu, and A. Bayrak, "An asymp-
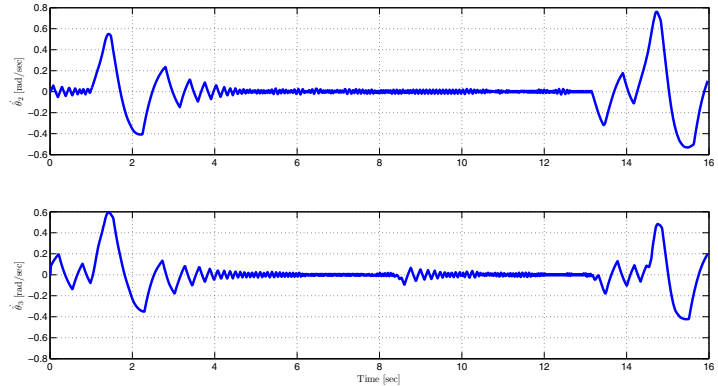

Fig. 7. Observed velocity $\dot{\hat{\theta}}(t)$
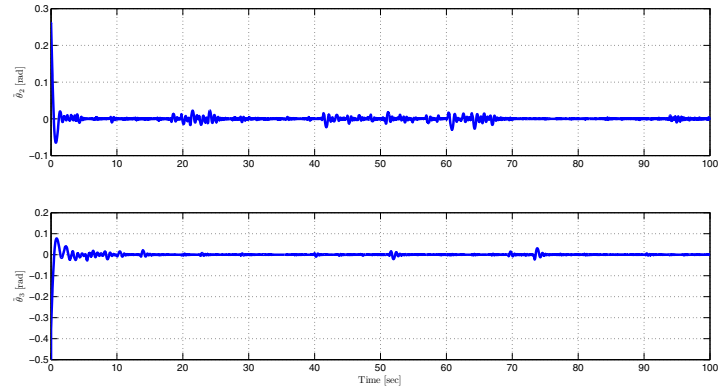

Fig. 8. Position observation errors $\tilde{\theta}(t)$

totically stable robust controller formulation for a class of MIMO nonlinear systems with uncertain dynamics," Int. J. of Systems Science, 2016, accepted, to appear, doi. 10.1080/00207721.2015.1039627.

[13] M. Krstic, Delay Compensation for Nonlinear, Adaptive, and PDE Systems. Boston, MA, USA: Birkhauser, 2009.

\section{APPENDIX I \\ PROOF OF LEMMA 1}

After substituting (12) into (15) and then integrating in time, the following expression is obtained

$$
\begin{aligned}
\int_{t_{0}}^{t} L_{1}(\sigma) d \sigma= & \int_{t_{0}}^{t} \tilde{x}^{T}(\sigma)\left[N(\sigma)-\beta_{1} \operatorname{Sgn}(\tilde{x}(\sigma))\right] d \sigma \\
& +\int_{t_{0}}^{t}\left[\frac{d \tilde{x}(\sigma)}{d \sigma}\right]^{T} N(\sigma) d \sigma \\
& -\int_{t_{0}}^{t}\left[\frac{d \tilde{x}(\sigma)}{d \sigma}\right]^{T} \beta_{1} \operatorname{Sgn}(\tilde{x}(\sigma)) d \sigma .
\end{aligned}
$$

After integrating the second integral on the right-hand side by parts and evaluating the third integral [13], following 

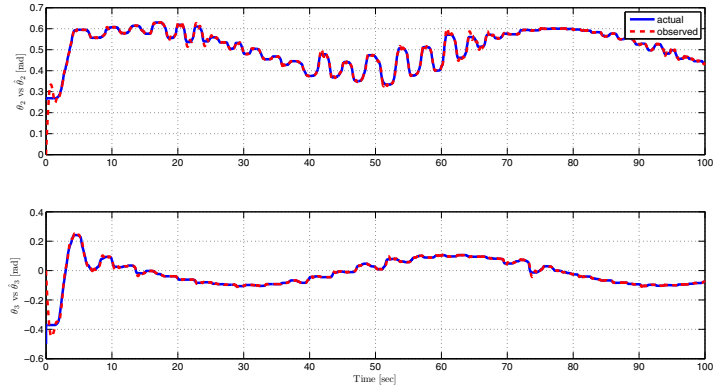

Fig. 9. Actual positions $\theta(t)$ (line) vs Observed positions $\hat{\theta}(t)$ (dashed)
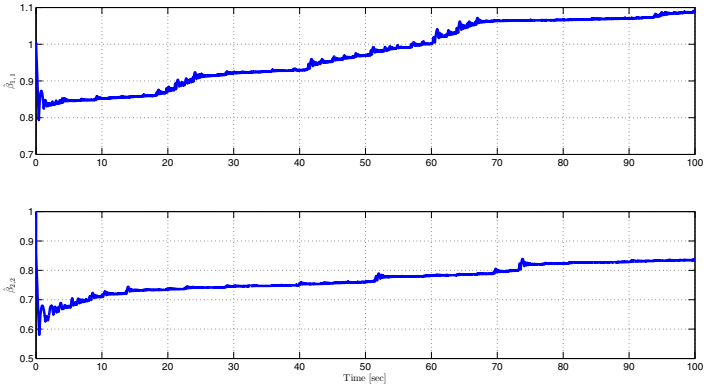

Fig. 10. Time varying gain $\hat{\beta}(t)$

expression is obtained

$$
\begin{aligned}
\int_{t_{0}}^{t} L_{1}(\sigma) d \sigma= & \int_{t_{0}}^{t} \tilde{x}^{T}(\sigma)\left[N(\sigma)-\beta_{1} \operatorname{Sgn}(\tilde{x}(\sigma))\right] d \sigma \quad \leq \sum_{i=1}^{m} \beta_{2_{i}}\left|\tilde{x}_{i}\left(t_{0}\right)\right| \\
& +\left.\left[\tilde{x}^{T}(\sigma) N(\sigma)\right]\right|_{t_{0}} ^{t}-\int_{t_{0}}^{t} \tilde{x}^{T}(\sigma) \frac{d N(\sigma)}{d \sigma} d \begin{array}{r}
\text { From (40), we can conclude that when the diagonal } \\
\text { of } \beta_{2} \text { are chosen as positive, then (19) holds with }
\end{array} \\
& -\left.\sum_{i=1}^{m} \beta_{1_{i}}\left|\tilde{x}_{i}(\sigma)\right|\right|_{t_{0}} ^{t} \\
= & \int_{t_{0}}^{t} \tilde{x}^{T}(\sigma)\left[N(\sigma)-\frac{d N(\sigma)}{d \sigma}-\beta_{1} \operatorname{Sgn}(\tilde{x}(\sigma))\right] d \sigma \\
& +\tilde{x}^{T}(t) N(t)-\sum_{b_{2}}^{m} \beta_{2_{i}}\left|\tilde{x}_{i}\left(t_{0}\right)\right| \\
& -\sum_{i=1}^{m} \beta_{1_{i}}\left|\tilde{x}_{i}(t)\right|+\sum_{i=1}^{m} \beta_{1_{i}}\left|\tilde{x}_{i}\left(t_{0}\right)\right| .
\end{aligned}
$$

\section{APPENDIX II}

PROOF OF LEMMA 2

After integrating (18) in time, following steps can be obtained [13]

$$
\begin{aligned}
\int_{t_{0}}^{t} L_{2}(\sigma) d \sigma & =-\int_{t_{0}}^{t} \dot{\tilde{x}}^{T}(\sigma) \beta_{2} \operatorname{Sgn}(\tilde{x}(\sigma)) d \sigma \\
& =-\int_{t_{0}}^{t}[\operatorname{Sgn}(\tilde{x})]^{T} \beta_{2} d(\tilde{x}) \\
& =-\sum_{i=1}^{m}\left(\beta_{2_{i}} \int_{t_{0}}^{t} d\left(\left|\tilde{x}_{i}\right|\right)\right) \\
& =-\sum_{i=1}^{m}\left[\beta_{2_{i}}\left(\left|\tilde{x}_{i}(t)\right|-\left|\tilde{x}_{i}\left(t_{0}\right)\right|\right)\right] \\
& \leq \sum_{i=1}^{m} \beta_{2_{i}}\left|\tilde{x}_{i}\left(t_{0}\right)\right| .
\end{aligned}
$$

The right-hand side of (37) can be upper bounded as

$$
\begin{aligned}
\int_{t_{0}}^{t} L_{1}(\sigma) d \sigma \leq & \sum_{i=1}^{m} \int_{t_{0}}^{t}\left|\tilde{x}_{i}(\sigma)\right|\left(\left|N_{i}(\sigma)\right|+\left|\frac{d N_{i}(\sigma)}{d \sigma}\right|-\beta_{1_{i}}\right) d \sigma \\
& +\sum_{i=1}^{m}\left|\tilde{x}_{i}(t)\right|\left(\left|N_{i}(t)\right|-\beta_{1_{i}}\right) \\
& +\sum_{i=1}^{m} \beta_{1_{i}}\left|\tilde{x}_{i}\left(t_{0}\right)\right|-\tilde{x}_{i}\left(t_{0}\right) N_{i}\left(t_{0}\right)
\end{aligned}
$$

From (38), we can claim that if the entries of $\beta_{1}$ satisfy (16), then (17) holds with

$$
\zeta_{b_{1}} \triangleq \sum_{i=1}^{m}\left[\beta_{1_{i}}\left|\tilde{x}_{i}\left(t_{0}\right)\right|-\tilde{x}_{i}\left(t_{0}\right) N_{i}\left(t_{0}\right)\right]
$$

